Barndorff-Nielsen, Stelzer:

Absolute Moments of Generalized Hyperbolic Distributions and Approximate Scaling of Normal Inverse Gaussian Lévy-Processes

Sonderforschungsbereich 386, Paper 381 (2004)

Online unter: http://epub.ub.uni-muenchen.de/

Projektpartner
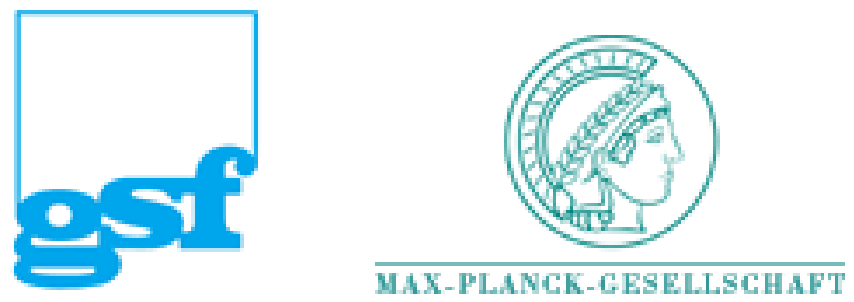


\title{
Absolute Moments of Generalized Hyperbolic Distributions and Approximate Scaling of Normal Inverse Gaussian Lévy-Processes
}

\author{
Ole Eiler Barndorff-Nielsen* $\quad$ Robert Stelzer ${ }^{\dagger}$
}

April 25, 2004

\begin{abstract}
Expressions for (absolute) moments of generalized hyperbolic (GH) and normal inverse Gaussian (NIG) laws are given in terms of moments of the corresponding symmetric laws. For the (absolute) moments centered at the location parameter $\mu$ explicit expressions as series containing Bessel functions are provided.

Furthermore the derivatives of the logarithms of (absolute) $\mu$-centered moments with respect to the logarithm of time are calculated explicitly for NIG Lévy processes. Computer implementation of the formulae obtained is briefly discussed. Finally some further insight into the apparent scaling behaviour of NIG Lévy processes (previously discussed in Barndorff-Nielsen and Prause (2001)) is gained.
\end{abstract}

Keywords: generalized inverse Gaussian distribution, high-frequency data in finance, normal inverse Gaussian distribution, scaling

AMS Subject Classification 2000: Primary: 60G51, 62E15

Secondary: 91B28

\footnotetext{
*Department of Mathematical Sciences, University of Århus, Ny Munkegade, DK-8000 Århus C, Denmark. oebn@imf .au.dk

${ }^{\dagger}$ Centre for Mathematical Sciences, Munich University of Technology, D-85747 Garching, Germany. rstelzer@ma.tum.de
} 


\section{Introduction}

In recent years many authors have successfully fitted generalized hyperbolic (GH) distributions and in particular normal inverse Gaussian (NIG) laws to returns in financial time series; see Eberlein and Keller (1995), Prause (1997), Barndorff-Nielsen (1997), Prause (1999), Barndorff-Nielsen and Shephard (2001) and references therein, Schoutens (2003), Barndorff-Nielsen and Shephard (2005). Moreover, BarndorffNielsen, Blæsild and Schmiegel (2004) recently demonstrated (following an indication in Barndorff-Nielsen (1998a)) that the NIG law is capable of describing velocity data from turbulence experiments with high accuracy.

This has, in particular, led to modeling the time dynamics of financial markets by stochastic processes using generalized hyperbolic or normal inverse Gaussian laws and associated Lévy processes as building blocks (e.g. Rydberg (1997), Bibby and Sørensen (1997), Barndorff-Nielsen (1998b), Rydberg (1999), Prause (1999), Raible (2000), Barndorff-Nielsen and Shephard (2001, 2005) and references therein, Barndorff-Nielsen (2001), Eberlein (2001), Schoutens (2003), Cont and Tankov (2004) and Emmer and Klüppelberg (2004)).

Empirical studies of financial data from the foreign exchange markets have regularly reported that the absolute moments of returns exhibit a power scaling behaviour, see e.g. Müller, Dacorogna, Olsen, Pictet, Schwarz and Morgenegg (1990) and Guillaume, Dacorogna, Davé, Müller, Olsen and Pictet (1997). Barndorff-Nielsen and Prause (2001) found that the power scaling coefficients obtained in these empirical studies are well within the range to be expected under a NIG Lévy process. In their article they solely studied the first absolute moment and obtained analytic results only in the case of a symmetric NIG Lévy process, i.e. when the parameter $\beta$ is zero (for the parametrization of the NIG law see below). In this paper we generalize their findings to the skewed case. We start by deriving formulae for the (absolute) moments of the generalized hyperbolic distribution in terms of mixed moments of the corresponding symmetric GH law. For $\mu$-centered (absolute) moments, i.e. moments centered at the location parameter $\mu$, we are able to give explicit formulae using Bessel functions. From these general formulae we will then, as special cases, obtain formulae for the moments of the NIG law, resp. NIG Lévy process. Analytic results for the approximate power scaling are then obtained, expressed as the derivative of the logarithm of the $\mu$-centered absolute moments of the NIG Lévy process with respect to the logarithm of time. Based upon this we derive some asymptotic scaling properties for $t \rightarrow \infty$ and 0 .

In the final sections we will discuss the numerical implementation of the formulae obtained and give a numerical example for the apparent power scaling present in NIG Lévy processes fitted to real financial data (the US-Dollar/Deutsch Mark exchange rate). 


\section{Generalized hyperbolic and inverse Gaussian distributions}

We consider the class of one-dimensional generalized hyperbolic (GH) distributions. The GH laws were introduced in Barndorff-Nielsen (1977) and Barndorff-Nielsen (1978a). For some recent results see Prause (1999), Eberlein (2001) and Eberlein and Hammerstein (2002). We denote the generalized hyperbolic distribution by $G H(\nu, \alpha, \beta, \mu, \delta)$. The probability density is given by:

$$
\begin{aligned}
p(x ; \nu, \alpha, \beta, \mu, \delta)= & \frac{\bar{\gamma}^{\nu} \bar{\alpha}^{1 / 2-\nu}}{\sqrt{2 \pi} \delta K_{\nu}(\bar{\gamma})}\left(1+\frac{(x-\mu)^{2}}{\delta^{2}}\right)^{\nu / 2-1 / 4} \\
& \cdot K_{\nu-1 / 2}\left(\bar{\alpha} \sqrt{1+\frac{(x-\mu)^{2}}{\delta^{2}}}\right) e^{\beta(x-\mu)}
\end{aligned}
$$

for $x \in \mathbb{R}$ where the parameters satisfy $\nu \in \mathbb{R}, 0 \leq|\beta|<\alpha, \mu \in \mathbb{R}, \delta \in \mathbb{R}_{>0}$, and $\gamma:=\sqrt{\alpha^{2}-\beta^{2}}, \bar{\alpha}:=\delta \alpha, \bar{\beta}:=\delta \beta, \bar{\gamma}:=\delta \gamma$.

Furthermore, $K_{\nu}(\cdot)$ denotes the modified Bessel function of the third kind and order $\nu \in \mathbb{R}$. For a comprehensive discussion of Bessel functions of complex arguments see Watson (1952). Jørgensen (1982) contains an appendix listing important properties of Bessel functions of the third kind and related functions. Most of these properties can also be found in standard reference books like Gradshteyn and Ryzhik (1965) or Bronstein, Semendjaev, Mühlig and Musiol (2000). For the following we need to know that $K_{\nu}$ is defined on the positive half plane $D=\{z \in \mathbb{C}: \Re(z)>0\}$ of the complex numbers and is holomorphic on $D$. From Watson (1952, p. 182) or Jørgensen (1982, p. 170) we have the representation

$$
K_{\nu}(z)=\frac{1}{2} \int_{0}^{\infty} y^{\nu-1} e^{-\frac{1}{2} z\left(y+y^{-1}\right)} d y
$$

which shows the strict positivity of $K_{\nu}$ on $\mathbb{R}_{>0}$. The substitution $x:=y^{-1}$ immediately gives $K_{-\nu}=K_{\nu}$. Furthermore, $K_{\nu}(z)$ is obviously monotonically decreasing in $z$ on $\mathbb{R}_{>0}$. From the alternative representation

$$
K_{\nu}(z)=\int_{0}^{\infty} e^{-z \cosh (t)} \cosh (\nu t) d t
$$

(cf. Watson $\left(1952\right.$, p. 181)) one reads off that, for fixed $z \in \mathbb{R}_{>0}, K_{\nu}(z)$ is strictly increasing in $\nu$ for $\nu \in \mathbb{R}_{\geq 0}$.

A very useful representation in law of the generalized hyperbolic distribution can be given using the generalized inverse Gaussian distribution. The generalized inverse Gaussian distribution $G I G(\nu, \delta, \gamma)$ with parameters $\nu \in \mathbb{R}, \gamma, \delta \in \mathbb{R}_{\geq 0}$ and $\gamma+\delta>0$ is the distribution on $\mathbb{R}_{>0}$ which has probability density function

$$
\begin{aligned}
p(x ; \nu, \delta, \gamma) & =\frac{(\gamma / \delta)^{\nu}}{2 K_{\nu}(\delta \gamma)} x^{\nu-1} \exp \left(-\frac{1}{2}\left(\delta^{2} x^{-1}+\gamma^{2} x\right)\right) \\
& =\frac{\bar{\gamma}^{\nu}}{2 K_{\nu}(\bar{\gamma})} \delta^{-2 \nu} x^{\nu-1} \exp \left(-\frac{1}{2}\left(\delta^{2} x^{-1}+\bar{\gamma}^{2} \delta^{-2} x\right)\right)
\end{aligned}
$$


For more information on the GIG law we refer to Jørgensen (1982) and for an interpretation in terms of hitting times to Barndorff-Nielsen, Blæsild and Halgreen (1978). The following normal variance-mean mixture representation of the generalized hyperbolic law holds.

Lemma 1 Let $X \sim G H(\nu, \alpha, \beta, \mu, \delta), V \sim G I G(\nu, \delta, \gamma)$ with $\gamma=\sqrt{\alpha^{2}-\beta^{2}}$ and $\varepsilon \sim N(0,1)$, where $V$ and $\varepsilon$ are independent, then:

$$
X \stackrel{\mathscr{D}}{=} \mu+\beta V+\sqrt{V} \varepsilon
$$

(For a general overview over normal variance-mean mixtures see Barndorff-Nielsen, Kent and Sørensen (1982).)

Furthermore, the cumulant function of the generalized hyperbolic law $X \sim G H(\nu$, $\alpha, \beta, \mu, \delta)$ is given by

$$
\mathrm{K}(\theta \ddagger X)=\frac{\nu}{2} \log \left(\frac{\gamma}{\alpha^{2}-(\beta+\theta)^{2}}\right)+\log \left(\frac{K_{\nu}\left(\delta \sqrt{\alpha^{2}-(\beta+\theta)^{2}}\right)}{K_{\nu}\left(\delta \sqrt{\alpha^{2}-\beta^{2}}\right)}\right)+\theta \mu .
$$

Obviously $\mathrm{K}(\theta \ddagger X)$ is defined for all $\theta \in \mathbb{R}$ with $|\beta+\theta|<\alpha$. From this fact and Barndorff-Nielsen (1978b, Corollary 7.1) we immediately obtain:

Lemma 2 Assume $X \sim G H(\nu, \alpha, \beta, \mu, \delta)$. Then $X \in L^{p}$ for all $p>0$, i.e. $E\left(|X|^{p}\right)$ exists for all $p>0$.

\section{Expressing moments via moments of sym- metric GH laws}

In this section we give expressions for different (absolute) moments of arbitrary GH distributions in terms of moments of corresponding symmetric GH distributions. Based upon this we obtain explicit expressions for $\mu$-centered (absolute) moments of GH distributions, employing the variance-mean mixture representation.

Theorem 3 Let $X \sim G H(\nu, \alpha, \beta, \mu, \delta), Y \sim G H(\nu, \alpha, 0, \mu, \delta)$, then for every $r>0$ and $n \in \mathbb{N}$ :

$$
\begin{aligned}
& \text { (i) } E\left(X^{n}\right)=\left(\frac{\bar{\gamma}}{\bar{\alpha}}\right)^{\nu} \frac{K_{\nu}(\bar{\alpha})}{K_{\nu}(\bar{\gamma})} \sum_{k=0}^{\infty} \frac{\beta^{k}}{k !} E\left(Y^{n}(Y-\mu)^{k}\right) \\
& \text { (ii) } E\left(|X|^{r}\right)=\left(\frac{\bar{\gamma}}{\bar{\alpha}}\right)^{\nu} \frac{K_{\nu}(\bar{\alpha})}{K_{\nu}(\bar{\gamma})} \sum_{k=0}^{\infty} \frac{\beta^{k}}{k !} E\left(|Y|^{r}(Y-\mu)^{k}\right) \\
& \text { (iii) } E\left((X-\mu)^{n}\right)=\left(\frac{\bar{\gamma}}{\bar{\alpha}}\right)^{\nu} \frac{K_{\nu}(\bar{\alpha})}{K_{\nu}(\bar{\gamma})} \sum_{k=0}^{\infty} \frac{\beta^{2 k+m}}{(2 k+m) !} E\left((Y-\mu)^{2 k+m+n}\right) \\
& \text { (iv) } E\left(|X-\mu|^{r}\right)=\left(\frac{\bar{\gamma}}{\bar{\alpha}}\right)^{\nu} \frac{K_{\nu}(\bar{\alpha})}{K_{\nu}(\bar{\gamma})} \sum_{k=0}^{\infty} \frac{\beta^{2 k}}{(2 k) !} E\left(|Y-\mu|^{2 k+r}\right),
\end{aligned}
$$

where $m:=n \bmod 2$. All moments above are finite. 
Note that from the cumulant function we have $E(Y)=\mu$ and $E(X)=\mu+$ $\beta \frac{\delta K_{\nu+1}(\bar{\gamma})}{\gamma K_{\nu}(\bar{\gamma})}$. Hence, we have that $E\left((Y-\mu)^{r}\right)$ are central moments, whereas $E((X-$ $\left.\mu)^{r}\right)$ are in general just $\mu$-centered moments. Note also that $\left.\operatorname{sgn} E\left((X-\mu)^{n}\right)\right)=\operatorname{sgn} \beta$ for all odd $n$.

Proof: We will only prove (ii), since the proofs of the other formulae proceed along the same lines, except that to obtain (iii) and (iv) one notes in the final step that odd central moments of $Y$ vanish, since the distribution of $Y$ is symmetric around $\mu$.

The series representation of the exponential function gives

$$
\begin{aligned}
E\left(|X|^{r}\right)= & \int_{\mathbb{R}} \frac{\bar{\gamma}^{\nu} \bar{\alpha}^{1 / 2-\nu}}{\sqrt{2 \pi} \delta K_{\nu}(\bar{\gamma})}\left(1+\frac{(x-\mu)^{2}}{\delta^{2}}\right)^{\nu / 2-1 / 4} \\
& \cdot K_{\nu-1 / 2}\left(\bar{\alpha} \sqrt{1+\frac{(x-\mu)^{2}}{\delta^{2}}}\right) e^{\beta(x-\mu)}|x|^{r} d x \\
= & \int_{\mathbb{R}} \sum_{k=0}^{\infty} \frac{\bar{\gamma}^{\nu} \bar{\alpha}^{1 / 2-\nu}}{\sqrt{2 \pi} \delta K_{\nu}(\bar{\gamma})}\left(1+\frac{(x-\mu)^{2}}{\delta^{2}}\right)^{\nu / 2-1 / 4} \\
& \cdot K_{\nu-1 / 2}\left(\bar{\alpha} \sqrt{1+\frac{(x-\mu)^{2}}{\delta^{2}}}\right) \frac{\beta^{k}}{k !}(x-\mu)^{k}|x|^{r} d x
\end{aligned}
$$

The integrals exist (cf. Lemma 2) and the same is true with $\beta$ changed to $-\beta$. This implies that the integrals

$$
\int_{\mu}^{\infty} \frac{\bar{\gamma}^{\nu} \bar{\alpha}^{1 / 2-\nu}}{\sqrt{2 \pi} \delta K_{\nu}(\bar{\gamma})}\left(1+\frac{(x-\mu)^{2}}{\delta^{2}}\right)^{\nu / 2-1 / 4} K_{\nu-1 / 2}\left(\bar{\alpha} \sqrt{1+\frac{(x-\mu)^{2}}{\delta^{2}}}\right) e^{|\beta(x-\mu)|}|x|^{r} d x
$$

and

$$
\int_{-\infty}^{\mu} \frac{\bar{\gamma}^{\nu} \bar{\alpha}^{1 / 2-\nu}}{\sqrt{2 \pi} \delta K_{\nu}(\bar{\gamma})}\left(1+\frac{(x-\mu)^{2}}{\delta^{2}}\right)^{\nu / 2-1 / 4} K_{\nu-1 / 2}\left(\bar{\alpha} \sqrt{1+\frac{(x-\mu)^{2}}{\delta^{2}}}\right) e^{|\beta(x-\mu)|}|x|^{r} d x
$$

and hence the integral

$$
\int_{\mathbb{R}} \frac{\bar{\gamma}^{\nu} \bar{\alpha}^{1 / 2-\nu}}{\sqrt{2 \pi} \delta K_{\nu}(\bar{\gamma})}\left(1+\frac{(x-\mu)^{2}}{\delta^{2}}\right)^{\nu / 2-1 / 4} K_{\nu-1 / 2}\left(\bar{\alpha} \sqrt{1+\frac{(x-\mu)^{2}}{\delta^{2}}}\right) e^{|\beta(x-\mu)|}|x|^{r} d x
$$

exist. Using the last one as majorant, Lebesgue's convergence theorem gives

$$
\begin{aligned}
E\left(|X|^{r}\right)= & \sum_{k=0}^{\infty} \int_{\mathbb{R}} \frac{\bar{\gamma}^{\nu} \bar{\alpha}^{1 / 2-\nu}}{\sqrt{2 \pi} \delta K_{\nu}(\bar{\gamma})}\left(1+\frac{(x-\mu)^{2}}{\delta^{2}}\right)^{\nu / 2-1 / 4} \\
& \cdot K_{\nu-1 / 2}\left(\bar{\alpha} \sqrt{1+\frac{(x-\mu)^{2}}{\delta^{2}}}\right) \frac{\beta^{k}}{k !}(x-\mu)^{k}|x|^{r} d x \\
= & \sum_{k=0}^{\infty} \frac{\beta^{k}}{k !}(\overline{\bar{\gamma}})^{\nu} \frac{K_{\nu}(\bar{\alpha})}{K_{\nu}(\bar{\gamma})} \int_{\mathbb{R}} \frac{\bar{\alpha}^{\nu} \bar{\alpha}^{1 / 2-\nu}}{\sqrt{2 \pi} \delta K_{\nu}(\bar{\alpha})}\left(1+\frac{(x-\mu)^{2}}{\delta^{2}}\right)^{\nu / 2-1 / 4} \\
& \cdot K_{\nu-1 / 2}\left(\bar{\alpha} \sqrt{1+\frac{(x-\mu)^{2}}{\delta^{2}}}\right)(x-\mu)^{k}|x|^{r} d x .
\end{aligned}
$$


From this we immediately conclude

$$
E\left(|X|^{r}\right)=\left(\frac{\bar{\gamma}}{\bar{\alpha}}\right)^{\nu} \frac{K_{\nu}(\bar{\alpha})}{K_{\nu}(\bar{\gamma})} \sum_{k=0}^{\infty} \frac{\beta^{k}}{k !} E\left(|Y|^{r}(Y-\mu)^{k}\right) .
$$

Corollary 4 Let $X \sim G H(\nu, \alpha, \beta, \mu, \delta), V \sim G I G(\nu, \delta, \alpha)$ and $\varepsilon \sim N(0,1)$ with $V$ and $\varepsilon$ independent, then for every $r>0$ and $n \in \mathbb{N}$ :

(i) $E\left((X-\mu)^{n}\right)=\left(\frac{\bar{\gamma}}{\bar{\alpha}}\right)^{\nu} \frac{K_{\nu}(\bar{\alpha})}{K_{\nu}(\bar{\gamma})} \sum_{k=0}^{\infty} \frac{\beta^{2 k+m}}{(2 k+m) !} E\left(V^{k+(m+n) / 2}\right) E\left(\epsilon^{2 k+m+n}\right)$

(ii) $E\left(|X-\mu|^{r}\right)=\left(\frac{\bar{\gamma}}{\bar{\alpha}}\right)^{\nu} \frac{K_{\nu}(\bar{\alpha})}{K_{\nu}(\bar{\gamma})} \sum_{k=0}^{\infty} \frac{\beta^{2 k}}{(2 k) !} E\left(V^{k+r / 2}\right) E\left(|\epsilon|^{2 k+r}\right)$

where $m:=n \bmod 2$.

Proof: Combine Theorem 3 with Lemma 1.

Note that we obtain the (absolute) moments of $X$ provided $\mu=0$ and the (absolute) central moments if $\beta=0$. For $\beta=0$ the above series are in fact just a single term or vanish completely.

Using the explicit expressions for the moments of GIG and normal laws, given in the appendix, it is now straightforward to obtain more explicit expressions for the $\mu$-centered (absolute) moments of GH laws.

Theorem 5 Let $X \sim G H(\nu, \alpha, \beta, \mu, \delta)$, then for every $r>0$ and $n \in \mathbb{N}$ :

$$
\begin{aligned}
& \text { (i) } E\left((X-\mu)^{n}\right)=\frac{2^{\left\lceil\frac{n}{2}\right\rceil} \bar{\gamma}^{\nu} \delta^{2\left\lceil\frac{n}{2}\right\rceil} \beta^{m}}{\sqrt{\pi} K_{\nu}(\bar{\gamma}) \bar{\alpha}^{\nu+\left\lceil\frac{n}{2}\right\rceil}} \sum_{k=0}^{\infty} \frac{2^{k} \bar{\beta}^{2 k} \Gamma\left(k+\left\lceil\frac{n}{2}\right\rceil+\frac{1}{2}\right)}{\bar{\alpha}^{k}(2 k+m) !} K_{\nu+k+\left\lceil\frac{n}{2}\right\rceil}(\bar{\alpha}) \\
& \text { (ii) } E\left(|X-\mu|^{r}\right)=\frac{2^{\frac{r}{2}} \bar{\gamma}^{\nu} \delta^{r}}{\sqrt{\pi} K_{\nu}(\bar{\gamma}) \bar{\alpha}^{\nu+\frac{r}{2}}} \sum_{k=0}^{\infty} \frac{2^{k} \bar{\beta}^{2 k} \Gamma\left(k+\frac{r}{2}+\frac{1}{2}\right)}{\bar{\alpha}^{k}(2 k) !} K_{\nu+k+\frac{r}{2}}(\bar{\alpha}),
\end{aligned}
$$

where $m:=n \bmod 2$.

Proof: Combine Corollary 4 with Lemmas 12 and 13 noting that $(n+m) \bmod 2=0$ and $(m+n) / 2=(n \bmod 2+n) / 2=\left\lceil\frac{n}{2}\right\rceil$.

The absolute convergence of the series on the right hand sides is obviously implied by the finiteness of $E\left((X-\mu)^{n}\right)$, resp. $E\left(|X-\mu|^{r}\right)$, and the positivity of all terms involved. Yet, one can also immediately give an analytic argument, which adds further insight into the convergence behaviour and is useful when one implements the above formulae on a computer (see section 6). Let $a_{k}:=\frac{2^{k} \bar{\beta}^{2 k} \Gamma\left(k+\frac{r}{2}+\frac{1}{2}\right)}{\bar{\alpha}^{k}(2 k) !} K_{\nu+k+\frac{r}{2}}(\bar{\alpha})$. From $K_{\nu}(z) \sim \sqrt{\frac{\pi}{2}} 2^{\nu} \nu^{\nu-\frac{1}{2}} e^{-\nu} z^{-\nu}$ for $\nu \rightarrow \infty$ (Ismail (1977), Jørgensen (1982, p. 171)) we obtain

$$
\frac{a_{k+1}}{a_{k}} \sim \frac{4 \bar{\beta}^{2}\left(k+\frac{r}{2}+\frac{1}{2}\right)\left(k+\nu+\frac{r}{2}\right)\left(1+\frac{1}{k+\nu+\frac{r}{2}}\right)^{k+\nu+\frac{r+1}{2}}}{\bar{\alpha}^{2} e(2 k+2)(2 k+1)} \stackrel{k \rightarrow \infty}{\rightarrow}\left(\frac{\bar{\beta}}{\bar{\alpha}}\right)^{2}<1
$$


and thus the quotient criterion from standard analysis implies absolute convergence. Lemma 9, which we later state when looking at NIG Lévy processes, and its proof add some more insight into the behaviour of the series.

As a side result of Theorem 5 we also obtain two identities for modified Bessel functions of the third kind.

Corollary 6 Let $x, y, z \in \mathbb{R}_{>0}$ s.t. $z=\sqrt{x^{2}-y^{2}}$ and $\nu \in \mathbb{R}$ then

$$
\begin{aligned}
& \text { (i) } K_{\nu}(z)=\frac{z^{\nu}}{x^{\nu}} \sum_{k=0}^{\infty} \frac{1}{2^{k} \cdot k !} \frac{y^{2 k}}{x^{k}} K_{\nu+k}(x) \\
& \text { (ii) } z K_{\nu}(z)+y^{2} K_{\nu+1}(z)=\frac{z^{\nu+1}}{x^{\nu}} \sum_{k=0}^{\infty} \frac{2 k+1}{2^{k} \cdot k !} \frac{y^{2 k}}{x^{k}} K_{\nu+k}(x) .
\end{aligned}
$$

Proof: Combine Theorem 5 with $E(X)=\frac{\delta \bar{\beta}}{\bar{\gamma}} \frac{K_{\nu+1}(\bar{\gamma})}{K_{\nu}(\bar{\gamma})}$ and $E\left(X^{2}\right)=\delta^{2}\left(\frac{K_{\nu+1}(\bar{\gamma})}{\bar{\gamma} K_{\nu}(\bar{\gamma})}+\right.$ $\left.\frac{\bar{\beta}^{2}}{\bar{\gamma}^{2}} \frac{K_{\nu+2}(\bar{\gamma})}{K_{\nu}(\bar{\gamma})}\right)$ for $X \sim G H(\nu, \alpha, \beta, 0, \delta)$ and use $\Gamma(n+1 / 2)=(2 n) ! \sqrt{\pi} /\left(2^{2 n} \cdot n !\right)$. Finally identify $x, y, z, \nu$ with $\bar{\alpha}, \bar{\beta}, \bar{\gamma}, \nu+1$.

\section{Moments of NIG laws}

A subclass of the generalized hyperbolic laws, which is closed under convolution, is formed by the normal inverse Gaussian laws. For an overview see especially Barndorff-Nielsen (1998b). The $N I G(\alpha, \beta, \mu, \delta)$ law with $0 \leq|\beta|<\alpha, \mu \in \mathbb{R}$ and $\delta \in \mathbb{R}_{>0}$ is the special case of the $G H(\nu, \alpha, \beta, \mu, \delta)$ law given by $\nu=\frac{1}{2}$. Hence we can use the above calculations for ( $\mu$-centered) moments.

Corollary 7 Let $X \sim N I G(\alpha, \beta, \mu, \delta), Y \sim N I G(\alpha, 0, \mu, \delta)$, then for every $r>0$ and $n \in \mathbb{N}$ :

(i) $E\left(X^{n}\right)=e^{\bar{\gamma}-\bar{\alpha}} \sum_{k=0}^{\infty} \frac{\beta^{k}}{k !} E\left(Y^{n}(Y-\mu)^{k}\right)$

(ii) $E\left(|X|^{r}\right)=e^{\bar{\gamma}-\bar{\alpha}} \sum_{k=0}^{\infty} \frac{\beta^{k}}{k !} E\left(|Y|^{r}(Y-\mu)^{k}\right)$

(iii) $E\left((X-\mu)^{n}\right)=\frac{2^{\left\lceil\frac{n}{2}\right\rceil+\frac{1}{2}} \delta^{2\left\lceil\frac{n}{2}\right\rceil} \beta^{m}}{\pi \bar{\alpha}^{\left\lceil\frac{n}{2}\right\rceil-\frac{1}{2}}} e^{\bar{\gamma}} \sum_{k=0}^{\infty} \frac{2^{k} \bar{\beta}^{2 k} \Gamma\left(k+\left\lceil\frac{n}{2}\right\rceil+\frac{1}{2}\right)}{\bar{\alpha}^{k}(2 k+m) !} K_{k+\left\lceil\frac{n}{2}\right\rceil-\frac{1}{2}}(\bar{\alpha})$

(iv) $E\left(|X-\mu|^{r}\right)=\frac{2^{\frac{r+1}{2}} \delta^{r}}{\pi \bar{\alpha} \frac{r-1}{2}} e^{\bar{\gamma}} \sum_{k=0}^{\infty} \frac{2^{k} \bar{\beta}^{2 k} \Gamma\left(k+\frac{r+1}{2}\right)}{\bar{\alpha}^{k}(2 k) !} K_{k+\frac{r-1}{2}}(\bar{\alpha})$,

where $m:=n \bmod 2$. All moments above are finite.

Proof: Follows immediately from Theorems 3 and 5 using $K_{\frac{1}{2}}(z)=K_{-\frac{1}{2}}(z)=$ $\sqrt{\frac{\pi}{2}} z^{-1 / 2} e^{-z}$ (see e.g. Jørgensen (1982, p. 170)). 
Formulae (iii) and $(i v)$ for $r$ equal to an even natural number can be given more explicitly using

$$
K_{n+\frac{1}{2}}(z)=K_{\frac{1}{2}}(z)\left(1+\sum_{i=1}^{n} \frac{(n+i) !}{i !(n-i) !} 2^{-i} z^{-i}\right)
$$

for all $n \in \mathbb{N}$ (see e.g. Jørgensen (1982, p. 170)). But in order to avoid making the above formulae even more complex, we omit this.

\section{Moments of NIG Lévy processes and their time-wise behaviour}

Based on the above results our aim now is to generalize the findings of BarndorffNielsen and Prause (2001) regarding the time-wise approximate scaling behaviour of NIG Lévy processes.

\subsection{Moments of NIG Lévy processes}

Let $Z(t), t \in \mathbb{R}_{>0}$, be the $N I G(\alpha, \beta, \mu, \delta)$ Lévy process, i.e. $Z(1) \sim N I G(\alpha, \beta, \mu, \delta)$. The generalized hyperbolic distribution is infinitely divisible and hence for every GH law there is a Lévy process having it as marginal distribution at time one. However, for GH Lévy processes we do, in general, not know the marginal distribution at an arbitrary time $t$, whereas for NIG Lévy processes we have $Z(t) \sim N I G(\alpha, \beta, t \mu, t \delta)$. For more background on NIG Lévy processes see in particular Barndorff-Nielsen (1998b). From our previous results we can immediately infer:

Corollary 8 Let $Z(t), t \in \mathbb{R}_{>0}$, be a $N I G(\alpha, \beta, \mu, \delta)$ Lévy process, then for every $r>0$ and $n \in \mathbb{N}$ :

$$
\begin{aligned}
\text { (i) } E\left((Z(t)-\mu t)^{n}\right)= & \frac{2^{\left\lceil\frac{n}{2}\right\rceil+\frac{1}{2}} \delta^{2\left\lceil\frac{n}{2}\right\rceil} \beta^{m}}{\pi \bar{\alpha}^{\left\lceil\frac{n}{2}\right\rceil-\frac{1}{2}}} e^{t \bar{\gamma}} \sum_{k=0}^{\infty} \frac{2^{k} \bar{\beta}^{2 k} \Gamma\left(k+\left\lceil\frac{n}{2}\right\rceil+\frac{1}{2}\right)}{\bar{\alpha}^{k}(2 k+m) !} t^{k+\left\lceil\frac{n}{2}\right\rceil+\frac{1}{2}} \\
& \cdot K_{k+\left\lceil\frac{n}{2}\right\rceil-\frac{1}{2}}(t \bar{\alpha}) \\
\text { (ii) } E\left(|Z(t)-\mu t|^{r}\right)= & \frac{2^{\frac{r+1}{2}} \delta^{r}}{\pi \bar{\alpha}^{\frac{r-1}{2}}} e^{t \bar{\gamma}} \sum_{k=0}^{\infty} \frac{2^{k} \bar{\beta}^{2 k} \Gamma\left(k+\frac{r+1}{2}\right)}{\bar{\alpha}^{k}(2 k) !} t^{k+(r+1) / 2} K_{k+\frac{r-1}{2}}(t \bar{\alpha})
\end{aligned}
$$

where $m:=n \bmod 2$.

The following lemma, which shows that the moments above are analytic functions of time, is later needed to calculate derivatives of log moments.

Lemma 9 Let $\bar{\alpha}>0,|\bar{\beta}|<\bar{\alpha}, 1<\epsilon<\bar{\alpha}^{2} /|\bar{\beta}|^{2}, \nu \in \mathbb{R}, r>0, n \in \mathbb{N}, m=n \bmod 2$, $D=\{z \in \mathbb{C}: \Re(z)>0,|z|<\epsilon \Re(z)\}$,

$$
f: D \rightarrow \mathbb{C}, z \mapsto \sum_{k=0}^{\infty} \frac{2^{k} \bar{\beta}^{2 k} \Gamma\left(k+\frac{r}{2}+\frac{1}{2}\right)}{\bar{\alpha}^{k}(2 k) !} z^{k+(r+1) / 2} K_{\nu+k+\frac{r}{2}}(z \bar{\alpha})
$$


and

$$
g: D \rightarrow \mathbb{C}, z \mapsto \sum_{k=0}^{\infty} \frac{2^{k} \bar{\beta}^{2 k} \Gamma\left(k+\left\lceil\frac{n}{2}\right\rceil+\frac{1}{2}\right)}{\bar{\alpha}^{k}(2 k+m) !} z^{k+\left\lceil\frac{n}{2}\right\rceil+\frac{1}{2}} K_{\nu+k+\left\lceil\frac{n}{2}\right\rceil}(z \bar{\alpha}) .
$$

Then both series are locally uniformly convergent and $f, g$ are holomorphic on $D$.

Note that $\nu$ is $-1 / 2$ in the series of Corollary 8.

Proof: It is sufficient to show the locally uniform convergence, since this implies the holomorphicity via Weierstraß's convergence theorem for sequences of holomorphic functions (see some standard book on complex function theory, e.g. Freitag and Busam (2000, p. 100)). Furthermore it is obvious that the result for $g$ follows from the one for $f$.

Let us now prove the uniform convergence of the series $f(z)=\sum_{k=0}^{\infty} f_{k}(z)$ on $D \cap\{z \in \mathbb{C}: a<\Re(z)<b\}$ for arbitrary $0<a<b<\infty$, where $f_{k}(z)=$ $\frac{2^{k} \bar{\beta}^{2 k} \Gamma\left(k+\frac{r}{2}+\frac{1}{2}\right)}{\bar{\alpha}^{k}(2 k) !} z^{k+(r+1) / 2} K_{\nu+k+\frac{r}{2}}(z \bar{\alpha})$. An immediate consequence of the integral representation for $K_{\nu}$ given in (2) is $\left|K_{\nu}(z)\right| \leq K_{\nu}(\Re(z))$ for $z \in D$ and thus

$$
\begin{aligned}
\left|f_{k}(z)\right| & =\left|\frac{2^{k} \bar{\beta}^{2 k} \Gamma\left(k+\frac{r}{2}+\frac{1}{2}\right)}{\bar{\alpha}^{k}(2 k) !} z^{k+(r+1) / 2} K_{\nu+k+\frac{r}{2}}(z \bar{\alpha})\right| \\
& \leq \quad \frac{2^{k}|\bar{\beta}|^{2 k} \Gamma\left(k+\frac{r}{2}+\frac{1}{2}\right)}{\bar{\alpha}^{k}(2 k) !}(\epsilon \Re(z))^{k+(r+1) / 2} K_{\nu+k+\frac{r}{2}}(\Re(z) \bar{\alpha}) \\
& \leq:=\Re(z) \\
& \leq \frac{2^{k}|\bar{\beta}|^{2 k} \Gamma\left(k+\frac{r}{2}+\frac{1}{2}\right)}{\bar{\alpha}^{k}(2 k) !}(\epsilon x)^{k+(r+1) / 2} K_{\nu+\frac{r}{2}+k}(x \bar{\alpha})
\end{aligned}
$$

for all $k \in \mathbb{N}_{0}$. Note that we defined $x \in(a, b)$ to be the real part of $z$. Using $K_{\nu}^{\prime}(z)=-K_{\nu-1}(z)-\nu z^{-1} K_{\nu}(z)$ (see e.g. Jørgensen (1982, p. 170) or Bronstein et al. (2000, p. 528)) we obtain

$$
\frac{d}{d x} x^{k+\nu+\frac{r}{2}} K_{\nu+\frac{r}{2}+k}(x \bar{\alpha})=-\bar{\alpha} x^{k+\nu+\frac{r}{2}} K_{\nu+\frac{r}{2}+k-1}(x \bar{\alpha})<0 .
$$

This implies for $x \in(a, b)$ :

$$
x^{k+(r+1) / 2} K_{\nu+\frac{r}{2}+k}(x \bar{\alpha}) \leq d a^{k+(r+1) / 2} K_{\nu+\frac{r}{2}+k}(a \bar{\alpha})
$$

where $d:=a^{\nu-\frac{1}{2}} \cdot \max \left\{a^{-\nu+\frac{1}{2}}, b^{-\nu+\frac{1}{2}}\right\}$. Applying this inequality to the above expression, we get for all $k \in \mathbb{N}_{0}$

$$
\left|f_{k}(z)\right| \leq \frac{2^{k} d|\bar{\beta}|^{2 k} \Gamma\left(k+\frac{r}{2}+\frac{1}{2}\right)}{\bar{\alpha}^{k}(2 k) !}(\epsilon a)^{k+(r+1) / 2} K_{\nu+\frac{r}{2}+k}(a \bar{\alpha}) .
$$

From the finiteness of the $\frac{r}{2}$ th absolute moment of the $G H(\nu, a \bar{\alpha}, a|\bar{\beta}| \sqrt{\epsilon}, 0,1)$ law and Theorem 5 follows that

$$
\begin{aligned}
& \sum_{k=0}^{\infty} \frac{2^{k} d|\bar{\beta}|^{2 k} \Gamma\left(k+\frac{r}{2}+\frac{1}{2}\right)}{\bar{\alpha}^{k}(2 k) !}(\epsilon a)^{k+(r+1) / 2} K_{\nu+\frac{r}{2}+k}(a \bar{\alpha}) \\
= & d(\epsilon a)^{\frac{r+1}{2}} \sum_{k=0}^{\infty} \frac{2^{k}(|\bar{\beta}| a \sqrt{\epsilon})^{2 k} \Gamma\left(k+\frac{r}{2}+\frac{1}{2}\right)}{(a \bar{\alpha})^{k}(2 k) !} K_{\nu+\frac{r}{2}+k}(a \bar{\alpha})
\end{aligned}
$$

converges absolutely. Hence, the uniform convergence of $\sum_{k=0}^{\infty}\left|f_{k}(z)\right|$ on $D \cap\{z \in$ $\mathbb{C}: a<\Re(z)<b\}$ is established. 


\subsection{Scaling and apparent scaling}

Before we now turn to discussing the scaling properties of an NIG Lévy process, let us briefly state what scaling precisely means. Let $X(t)$ be some stochastic process. We say some moment of $X$ obeys a scaling law, if the logarithm of this moment is an affine function of $\log$ time i.e., for the $r$-th absolute moment, $\ln E\left(|X(t)|^{r}\right)=s \ln t+c$ for some constants $s, c \in \mathbb{R}$. Here $s$ is called the scaling coefficient. If all (absolute) moments of $X$, or at least those one is interested in, follow a scaling law, we say that the process itself obeys one. For example in the case of Brownian motion $X(t)$ with drift $\mu$ we know from $X(t)-\mu t \stackrel{\mathscr{D}}{=} \sqrt{t}(X(1)-\mu)$ that $\ln E\left(|X(t)-\mu t|^{r}\right)=$ $\frac{r}{2} \ln t+$ constant for all $r>0$, i.e. all absolute moments exhibit scaling. More generally all selfsimilar processes, e.g. the strictly $\alpha$-stable Lévy processes (cf. Samorodnitsky and Taqqu (1994, chapter 7) and Sato (1999, chapter 3)), obey a scaling law. When looking only at small changes in time the local scaling behaviour is determined by $\frac{d \ln E\left(|X(t)|^{r}\right)}{d \ln t}$ (in the case of the $r$-th absolute moment). In the presence of scaling the latter derivative is constant and equals the value of the scaling coefficient. Provided some log moment of a process $X(t)$ exhibits a very close to affine dependence on $\log$ time over some time horizon of interest, we speak of approximate or apparent scaling. This is equivalent to the local scaling behaviour varying only little over the time spans considered. When working with real empirical data, it is often not possible to distinguish between apparent and strict scaling due to the randomness of the available observations. Hence, it may be of interest, from a statistical point of view, whether some given process shows approximate scaling.

\subsection{The time-wise behaviour of $\mu$-centered moments}

Let us now examine the scaling behaviour exhibited by the $N I G(\alpha, \beta, \mu, \delta)$ Lévy process $Z(t)$. For the following discussion of the time dependence of $E\left(|Z(t)-\mu t|^{r}\right)$ we will abbreviate the time independent terms:

$$
\begin{aligned}
c(r) & :=\frac{2^{\frac{r+1}{2}} \delta^{r}}{\pi \bar{\alpha}^{\frac{r-1}{2}}} \\
a_{k}(r) & :=\frac{2^{k} \bar{\beta}^{2 k} \Gamma\left(k+\frac{r+1}{2}\right)}{\bar{\alpha}^{k}(2 k) !}
\end{aligned}
$$

If we define

$$
\psi(t):=\exp (t \bar{\gamma}) \sum_{k=0}^{\infty} a_{k}(r) t^{k+(r+1) / 2} K_{k+(r-1) / 2}(t \bar{\alpha})
$$

and

$$
\phi(t):=\ln \psi\left(e^{t}\right)
$$

we have from Corollary 8 that

$$
E\left(|Z(t)-\mu t|^{r}\right)=c(r) \cdot \psi(t)
$$


and

$$
\ln E\left(|Z(t)-\mu t|^{r}\right)=\ln c(r)+\phi(\ln t) .
$$

Thus:

$$
\frac{d \ln E\left(|Z(t)-\mu t|^{r}\right)}{d \ln t}=\phi^{\prime}(\ln t)
$$

Lemma 10 Let $\phi: \mathbb{R}_{>0} \rightarrow \mathbb{R}$ be defined by (11), then

$$
\phi^{\prime}(t)=1+\bar{\gamma} e^{t}-\bar{\alpha} e^{t} \frac{\sum_{k=0}^{\infty} a_{k}(r) e^{t k} K_{k+(r-3) / 2}\left(e^{t} \bar{\alpha}\right)}{\sum_{k=0}^{\infty} a_{k}(r) e^{t k} K_{k+(r-1) / 2}\left(e^{t} \bar{\alpha}\right)} .
$$

Proof: Using again $K_{\nu}^{\prime}(z)=-K_{\nu-1}(z)-\nu z^{-1} K_{\nu}(z)$, we obtain for $\psi(t)$ as defined in equation (10):

$$
\begin{aligned}
\psi^{\prime}(t)= & \exp (t \bar{\gamma})\left(\bar{\gamma} \sum_{k=0}^{\infty} a_{k}(r) t^{k+(r+1) / 2} K_{k+(r-1) / 2}(t \bar{\alpha})+\sum_{k=0}^{\infty} a_{k}(r)\left(k+\frac{r+1}{2}\right)\right. \\
& \cdot t^{k+(r-1) / 2} K_{k+(r-1) / 2}(t \bar{\alpha})-\sum_{k=0}^{\infty} a_{k}(r) t^{k+(r+1) / 2} \bar{\alpha} \\
& \left.\cdot\left(K_{k+(r-3) / 2}(t \bar{\alpha})+\left(k+\frac{r-1}{2}\right)(t \bar{\alpha})^{-1} K_{k+\frac{r-1}{2}}(t \bar{\alpha})\right)\right) \\
= & \bar{\gamma} \psi(t)+t^{-1} \psi(t)-\bar{\alpha} e^{\bar{\gamma} t} \sum_{k=0}^{\infty} a_{k}(r) t^{k+(r+1) / 2} K_{k+(r-3) / 2}(t \bar{\alpha}) .
\end{aligned}
$$

That we may interchange differentiation and summation above is an immediate consequence of Lemma 9 and Weierstraß's theorem for sequences of holomorphic functions (see e.g. Freitag and Busam (2000, p. 100)). Hence, we get from (11)

$$
\phi^{\prime}(t)=\frac{e^{t} \psi^{\prime}\left(e^{t}\right)}{\psi\left(e^{t}\right)}=1+\bar{\gamma} e^{t}-\bar{\alpha} e^{t} \frac{\sum_{k=0}^{\infty} a_{k}(r) e^{t(k+(r+1) / 2)} K_{k+(r-3) / 2}\left(e^{t} \bar{\alpha}\right)}{\sum_{k=0}^{\infty} a_{k}(r) e^{t(k+(r+1) / 2)} K_{k+(r-1) / 2}\left(e^{t} \bar{\alpha}\right)} .
$$

Now we can formulate our main result on the scaling behaviour of NIG-LévyProcesses.

Theorem 11 Let $Z(t), t \in \mathbb{R}_{>0}$, be a $N I G(\alpha, \beta, \mu, \delta)$ Lévy process, then

$$
\frac{d \ln E\left(|Z(t)-\mu t|^{r}\right)}{d \ln t}=1+\bar{\gamma} t-\bar{\alpha} t \frac{\sum_{k=0}^{\infty} a_{k}(r) t^{k} K_{k+(r-3) / 2}(\bar{\alpha} t)}{\sum_{k=0}^{\infty} a_{k}(r) t^{k} K_{k+(r-1) / 2}(\bar{\alpha} t)}
$$

for every $r>0$. 
Proof: The result follows by combining Lemma 10 and (14).

When comparing the above results with Barndorff-Nielsen and Prause (2001) note that they looked at the derivatives with respect to $\ln (\bar{\alpha} t)$, whereas we look at the derivative with respect to $\ln t$. The difference is related to the fact that BarndorffNielsen and Prause (2001) only consider the case $\beta=0$. In the general case the parameters $\bar{\alpha}$ and $\bar{\beta}$ of the marginals at time $t$ are both scaled with $t$ when the process evolves over time and hence it is most natural and convenient to consider the change of the log moments versus the change of log time directly.

The expression for the local scaling behaviour derived in Theorem 11 is in general not constant in time, hence the absolute $\mu$-centered moments of a NIG Lévy process do not obey a strict scaling law. Later we shall see from numerical examples that apparent scaling is common. If we look at the symmetric NIG Lévy process, i.e. $\beta=0$, the above formula becomes

$$
\frac{d \ln E\left(|Z(t)-\mu t|^{r}\right)}{d \ln t}=1+\bar{\alpha} t-\bar{\alpha} t \frac{K_{(r-3) / 2}(\bar{\alpha} t)}{K_{(r-1) / 2}(\bar{\alpha} t)} .
$$

From this one deducts using $K_{\nu}=K_{-\nu}$ that the second $\mu$-centered moment obeys a scaling law with slope one, which is the same as for Brownian motion. Yet, also in the symmetric case the $\mu$-centered absolute moments show no scaling behaviour in general.

The aggregational Gaussianity of NIG Lévy processes (cf. e.g. Barndorff-Nielsen and Shephard (2001)) becomes visible in the asymptotic scaling of the symmetric case for large times. For $r=1$ it was already noted in Barndorff-Nielsen and Prause (2001) that the local scaling approaches $1 / 2$ for $t \rightarrow \infty$ and hence for large $t$ the first absolute $\mu$-centered moment of the process seems to scale like Brownian motion. Using formula (ii) in Corollary 8 , which for $\beta=0$ becomes

$$
E\left(|Z(t)-\mu t|^{r}\right)=\frac{2^{\frac{r+1}{2}} \delta^{r}}{\pi \bar{\alpha}^{\frac{r-1}{2}}} \exp (t \bar{\alpha}) \Gamma\left(\frac{r+1}{2}\right) t^{(r+1) / 2} K_{\frac{r-1}{2}}(t \bar{\alpha}),
$$

and $K_{\nu}(x) \sim \sqrt{\pi / 2} x^{-1 / 2} e^{-x}$ for $x \rightarrow \infty$ (cf. Jørgensen (1982, p. 171) or Bronstein et al. (2000)) we get that $\ln E\left(|Z(t)-\mu t|^{r}\right)$ tends to $\frac{r}{2} \ln t+c$ for $t \rightarrow \infty$, where $c \in \mathbb{R}$ is a constant. Hence, $E\left(|Z(t)-\mu t|^{r}\right)$ obeys a scaling law with slope $r / 2$ for $t \rightarrow \infty$, i.e. the symmetric NIG Lévy process approaches the exact scaling behaviour of Brownian motion. This result can also be easily deduced from (16) using an asymptotic expansion of $\frac{K_{(r-3) / 2}(z)}{K_{(r-1) / 2}(z)}$ for $z \rightarrow+\infty$ (see e.g. Jørgensen (1982, p. 173)).

Studying the limiting behaviour of the absolute $\mu$-centered moments analytically for $\beta \neq 0$ seems hardly possible. Yet, numerical studies indicate that a skewed NIG Lévy process does not scale like Brownian motion for large times in general. For example when computing $\frac{d \ln E\left((Z(t))^{2}\right)}{d \ln t}$ of the $N I G(100,30,0,0.001)$ Lévy process for times from $1 / 2$ to 1024 the values increase monotonically from 1.004 to 6.268 . Note, however, that the numerical data presented in section 7 seems to indicate that for small values of $|\beta|$ (relative to $\alpha$ ) the value of $\frac{d \ln E\left(|Z(t)-\mu t|^{r}\right)}{d \ln t}$ may converge to something at least very close to $r / 2$, i.e. the moments scale very much like the ones of Brownian motion for large times. 
To see from (17) what happens in the symmetric case for $t \searrow 0$ we employ the fact that

$$
K_{\nu}(x) \sim \begin{cases}\Gamma(\nu) 2^{\nu-1} x^{-\nu} & \text { for } \nu>0, x \searrow 0 \\ -\ln x & \text { for } \nu=0, x \searrow 0\end{cases}
$$

(see e.g. Jørgensen (1982, p. 171)). For $r=1$ and $t \searrow 0$ we obtain that $\ln E(\mid Z(t)-$ $\mu t \mid)$ becomes $\ln t+\bar{\alpha} e^{\ln t}+\ln (-\ln (t \bar{\alpha}))+c$ with $c \in \mathbb{R}$ being a constant. From this we conclude that for small values of $t$ the first absolute $\mu$-centered moment approximately scales with slope one, as already noted in Barndorff-Nielsen and Prause (2001). The same asymptotic scaling slope of one holds for $r>1$, since $\ln E\left(|Z(t)-\mu t|^{r}\right) \sim$ $\ln t+\bar{\alpha} e^{\ln t}+c(r)$ for $t \searrow 0$. Yet, a different result is obtained for $0<r<1$. In this case $\ln E\left(|Z(t)-\mu t|^{r}\right) \sim r \ln t+\bar{\alpha} e^{\ln t}+c(r)$ and so there is asymptotic scaling with slope $r$.

\section{Notes on the numerical implementation}

We will now briefly discuss some issues related to the implementation of formula (ii) in Corollary 8 and Theorem 11 on a computer. Similar results hold for formula $(i)$ of Corollary 8. First note that (ii) in Corollary 8 can be reexpressed using (9) as:

$$
E\left(|Z(t)-\mu t|^{r}\right)=\left(\frac{2 \delta^{2} t}{\bar{\alpha}}\right)^{r / 2} \frac{\sqrt{2 t \bar{\alpha}}}{\pi} \exp (t \bar{\gamma}) \sum_{k=0}^{\infty} a_{k}(r) t^{k} K_{k+\frac{r-1}{2}}(t \bar{\alpha}) .
$$

The value of the infinite series can only be approximated. Yet, note that the analytic convergence discussion of the series in section 3, especially formula (6), implies asymptotically geometric convergence, which is the faster, the smaller $|\beta|$ is relatively to $\alpha$. We suggest to compute the individual summands recursively as discussed below, add them up and stop, when summands become negligible compared to the current value of the approximation. To calculate the individual summands recursively note that

$$
a_{k}(r) t^{k}=\frac{2 \bar{\beta}^{2}(k+(r-1) / 2)}{\bar{\alpha}(2 k-1)(2 k)} t \cdot a_{k-1}(r) t^{k-1}
$$

using the functional equation $\Gamma(z+1)=z \Gamma(z)$ of the Gamma function,

$$
a_{0}(r)=\Gamma\left(\frac{r+1}{2}\right)
$$

and

$$
K_{k+\frac{r-1}{2}}(t \bar{\alpha})=2 \cdot\left(k-1+\frac{r-1}{2}\right)(t \bar{\alpha})^{-1} K_{k-1+\frac{r-1}{2}}(t \bar{\alpha})+K_{k-2+\frac{r-1}{2}}(t \bar{\alpha}),
$$

see e.g. Jørgensen (1982, p. 170) or Bronstein et al. (2000, p. 528). The latter formula implies that we can calculate the values of the Bessel functions needed from a two term recursion, for which we only need to calculate $K_{-1+\frac{r-1}{2}}(t \bar{\alpha})$ and $K_{\frac{r-1}{2}}(t \bar{\alpha})$ as starting values. Hence, the calculation of the value of the series involves, apart 
from basic manipulations, only one evaluation of the Gamma function and two of Bessel functions.

The series in the denominator in Theorem 11 is the series just discussed above and the numerator is of the same type, only the index of the Bessel functions is changed, and can hence be calculated analogously. Actually, both series can be calculated simultaneously using only the recursion for $a_{k}(r) t^{k}$ and the two term recursion for the Bessel functions described above.

There is, however, one possible problem when using the two term recursion. If the starting values are zeros up to numerical precision, then only zeros will be calculated as summands. For example when using Matlab and the built in function for $K_{\nu}$ one gets $K_{0}(z)=0$ for $z>697$. Hence, one needs to take care of this possible case. Provided the recursion works, the numerical results obtained are usually almost identical to the numerical results one gets when using a built in Bessel function routine of e.g. Matlab for each summand, but the recursion saves computing power. Furthermore, it should now be obvious, how numerical evaluations of the formulae for $\mu$-centered (absolute) moments of GH laws given in Theorem 5 can be organized efficiently.

The Matlab code we used to produce the numerical results in this paper is available from www . ma.tum. de/stat/software. It is based upon the above considerations and can be used to compute $\mu$-centered moments of the NIG distribution/Lévy process and the derivatives of the log moments with respect to log time.

\section{Apparent scaling behaviour of NIG Lévy pro- cesses}

To exemplify the apparent scaling of general absolute $\mu$-centered moments of NIG Lévy processes, as in Barndorff-Nielsen and Prause (2001), we use the USD/DEM exchange rate returns from the whole of 1996, contained in the HFDF96 data set from Olsen \& Associates, and consider the NIG Lévy process $Z(t) \sim N I G(\alpha, \beta, t \mu, t \delta)$, $t \in \mathbb{R}_{>0}$, such that $Z(1) \sim N I G(\alpha, \beta, \mu, \delta)$, where the parameters are obtained by maximum likelihood estimation based on the three hour log returns. The estimates obtained are $\alpha=415.9049, \beta=1.512, \delta=0.0011$ and $\mu=0.000026$. Note especially that, as typical for returns of exchange rate series, $\mu$ is very close to zero and therefore there is practically no difference between moments and $\mu$-centered moments. Figure 1 (left), which depicts the logarithm of the first absolute $\mu$-centered moment versus the logarithm of time in seconds, is therefore optically indistinguishable from the figure in Barndorff-Nielsen and Prause (2001) showing the first absolute moment calculated via numerical integration. The estimated regression line of the log moments against log time, fitted by least squares, has slope 0.5863, which is slightly higher than the

slope reported in Barndorff-Nielsen and Prause (2001), and $\frac{d \ln E(|Z(t)-\mu t|)}{d \ln t}$ decreases from 0.7853 to 0.5011 over the time interval depicted, which is $5 \frac{5}{8}$ minutes to 32 days. The regression line slope of 0.5863 fits in well with the empirical results of Müller et al. (1990) and Guillaume et al. (1997), who report a scaling law with coefficient 0.58 to be typical for foreign exchange returns. This is significantly different from 

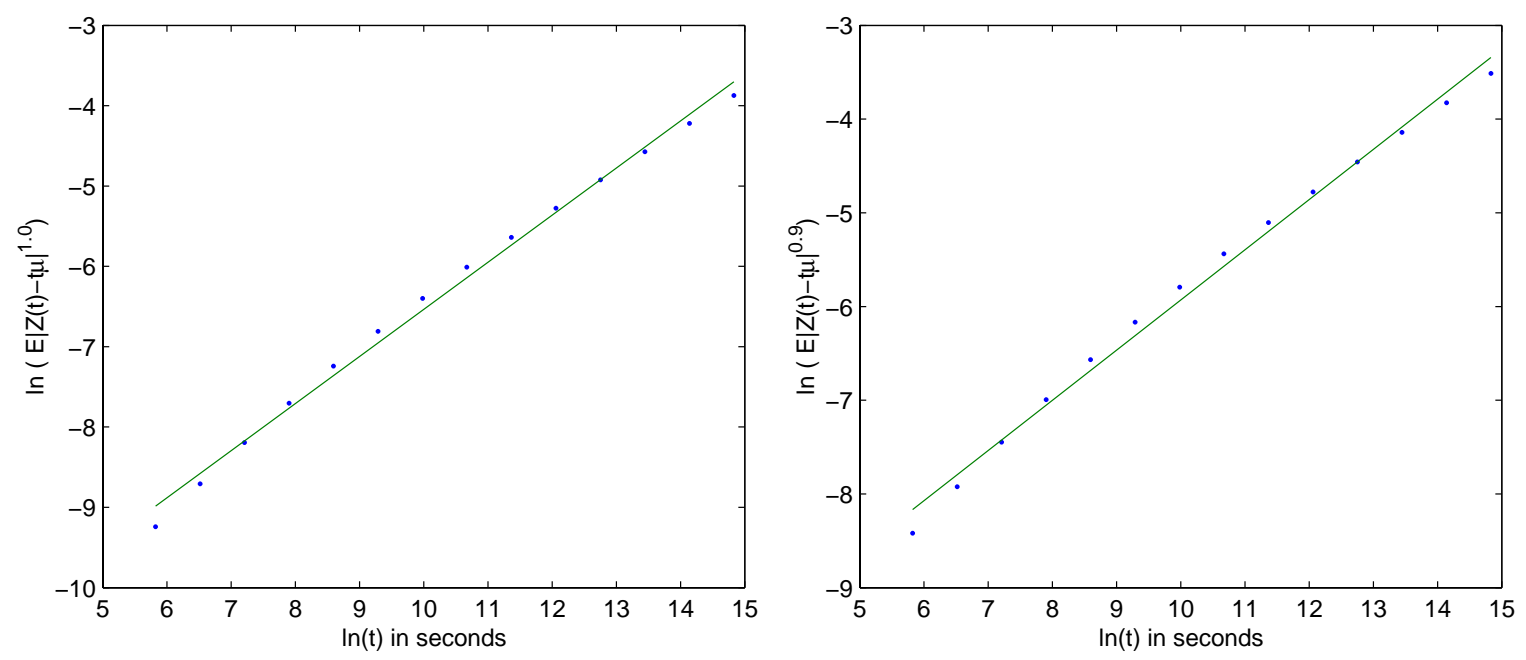

Figure 1: Approximate scaling power law for the first (left) and 0.9th $\mu$-centered absolute moment of the NIG Lévy process fitted to the USD/DEM exchange rate:

theoretical moments $(\bullet)$ and regression line log moments against log time

the Brownian motion case, where it is exactly $1 / 2$ (cf. above). The behaviour of $\frac{d \ln E(|Z(t)-\mu t|)}{d \ln t}$ over the time interval considered indicates that for $t \rightarrow \infty$ the slope asymptotically becomes about $1 / 2$, the exact Gaussian scaling coefficient. This is related to the fact that $|\beta|$ is relatively small, as already pointed out earlier in the discussion of the scaling asymptotics.

With our results obtained above it is possible to study the behaviour of moments other than the first. Figures 1 (right), 2 (left), 2 (right) and 3 (left) show the time behaviour of the 0.9 th, 1.1 th, 0.5 th and 1.5 th $\mu$-centered absolute moments over the same time horizon. All figures exhibit apparent scaling, which improves with the order of the moment. The fitted regression lines have slope $0.53535,0.63536$, 0.31322 and 0.81327 respectively, which are all higher than the corresponding values for Brownian motion 0.45, 0.55, 0.25 and 0.75. The values of $\frac{d \ln E\left(|Z(t)-\mu t|^{r}\right)}{d \ln t}$ decrease from 0.7316 to $0.4509,0.8316$ to $0.5512,0.4499$ to 0.2503 and 0.9499 to 0.7515 respectively. So again they seem to converge to some value around the Brownian motion scaling slope.

Figure 3 (right) shows that the second $\mu$-centered moment seems to exhibit perfect linear scaling. Yet, there is in fact no strict scaling law holding. The values of the regression coefficient 1.0001 and $\frac{d \ln E\left(|Z(t)-\mu t|^{2}\right)}{d \ln t}$ are very close to one with $\frac{d \ln E\left(|Z(t)-\mu t|^{2}\right)}{d \ln t}$ increasing very slowly from 1 to 1.0015 . Such a result is to be expected, since $|\beta|$ is small (compared to $\alpha$ ) and for $\beta=0$ we have that the variance, which is in this case identical to the second $\mu$-centered moment, obeys a strict scaling law with slope one as for Brownian motion.

The third $\mu$-centered absolute moment, see Figure 4 (left), still exhibits apparent scaling behaviour with a regression slope of 1.2966 , but the values of $\frac{d \ln E\left(|Z(t)-\mu t|^{3}\right)}{d \ln t}$ 

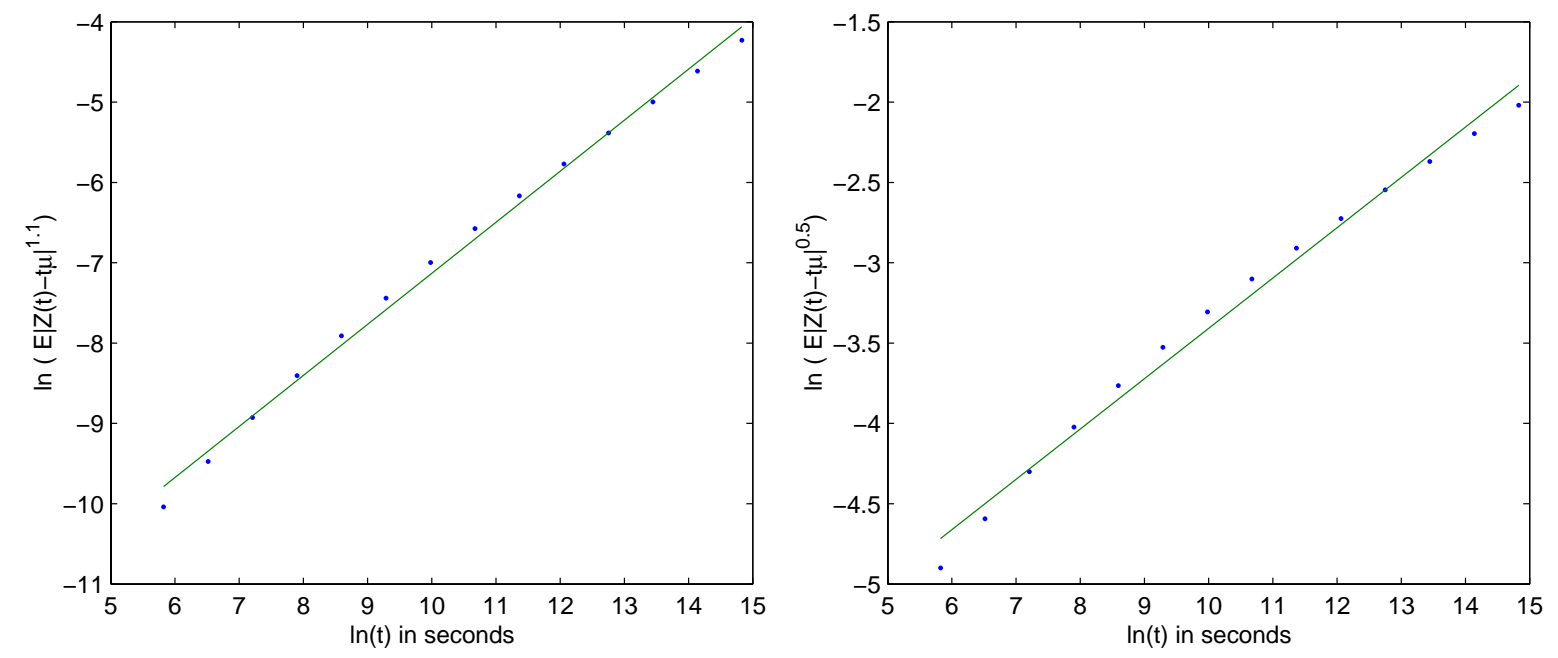

Figure 2: Approximate scaling power law for the 1.1th (left) and 0.5th $\mu$-centered absolute moment: theoretical moments $(\bullet)$ and regression line log moments against log time
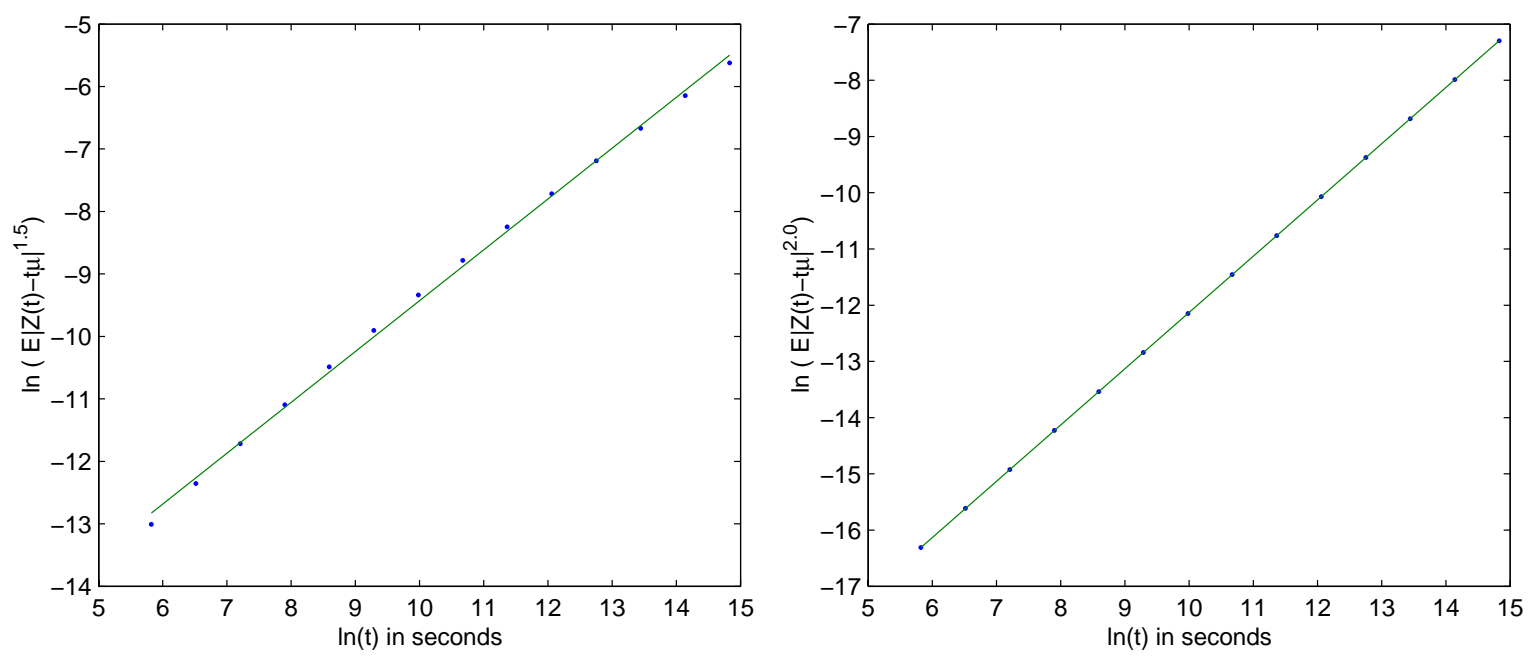

Figure 3: Approximate scaling power law for the 1.5th (left) and second $\mu$-centered absolute moment: theoretical moments $(\bullet)$ and regression line log moments against log time 

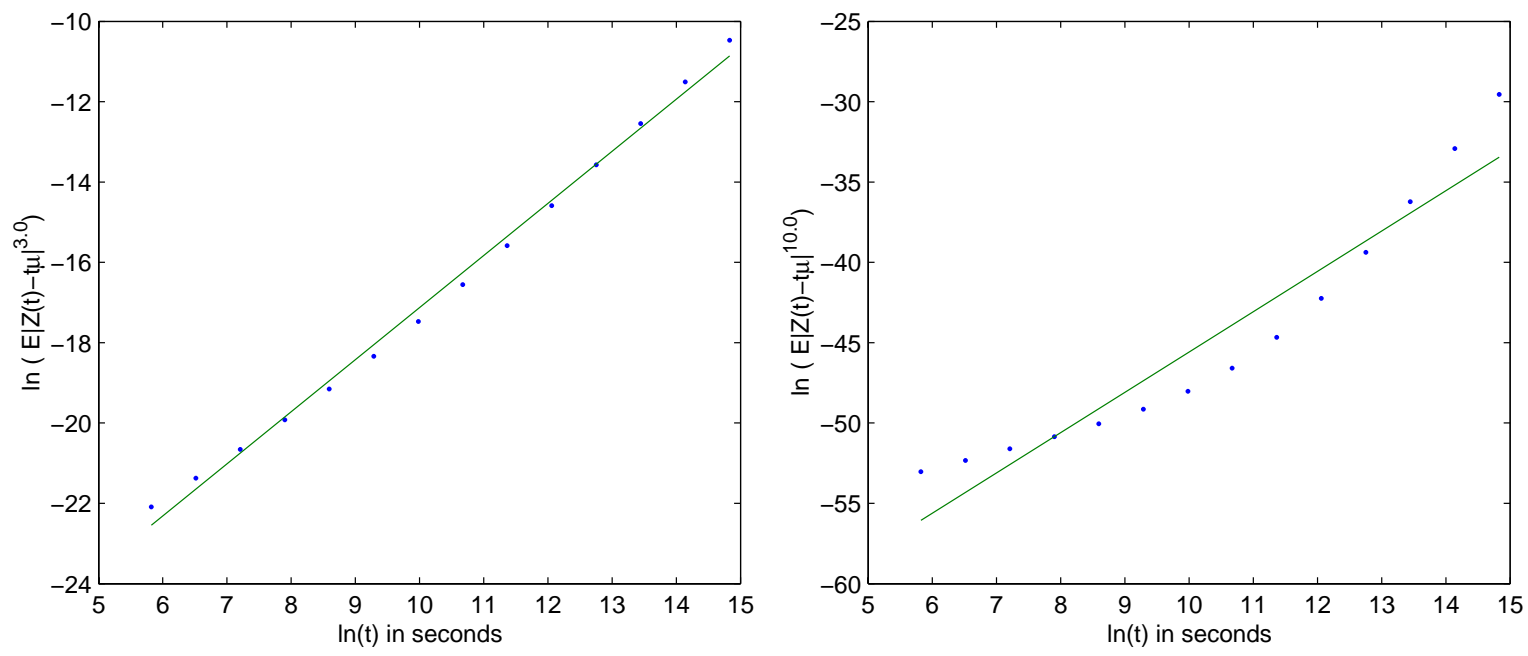

Figure 4: Approximate scaling power law for the third (left) and 10th $\mu$-centered absolute moment: theoretical moments $(\bullet)$ and regression line log moments against log time

are now increasing from 1.0134 to 1.5007 rather than decreasing and the slope is lower than the scaling coefficient 1.5 for Brownian motion. However, $\frac{d \ln E\left(|Z(t)-\mu t|^{3}\right)}{d \ln t}$ still seems to converge to some value close to $3 / 2$ at large times. It generally seems to be the case that $\frac{d \ln E\left(|Z(t)-\mu t|^{r}\right)}{d \ln t}$ increases with time for $r>2$, whereas it decreases for $r<2$. Actually further calculations indicate that this change takes place marginally below 2 at about 1.9995. Some more numerical calculations hint that in the symmetric case $\ln E\left(|Z(t)-\mu t|^{r}\right)$ is concave as a function of $\ln t$ for $0<r \leq 2$ and convex for $r \geq 2$.

For very high values of $r$ no apparent linear scaling is observed over the time horizon considered, see Figure 4 (right) for $r=10$ as an example.

Looking at the slopes of the apparent scaling of the $\mu$-centered absolute moments of orders 0.2 to 4 as plotted in Figure 5, the relationship between scaling coefficient and order is apparently not simply a linear one as, for example, in the case of an $\alpha$-stable Lévy process (see Samorodnitsky and Taqqu (1994, chapter 7) and Sato (1999, chapter 3)).

Our results obtained above, show that NIG Lévy processes are able to explain the stylized feature of scaling laws encountered in foreign exchange returns. Compared to Brownian motion the NIG Lévy process does in general not exhibit exact linear scaling, but apparent scaling over time horizons relevant in financial markets is common. The symmetric NIG Lévy process has the same exact scaling rule for the second $\mu$-centered moment, that is the variance, as Brownian motion. 

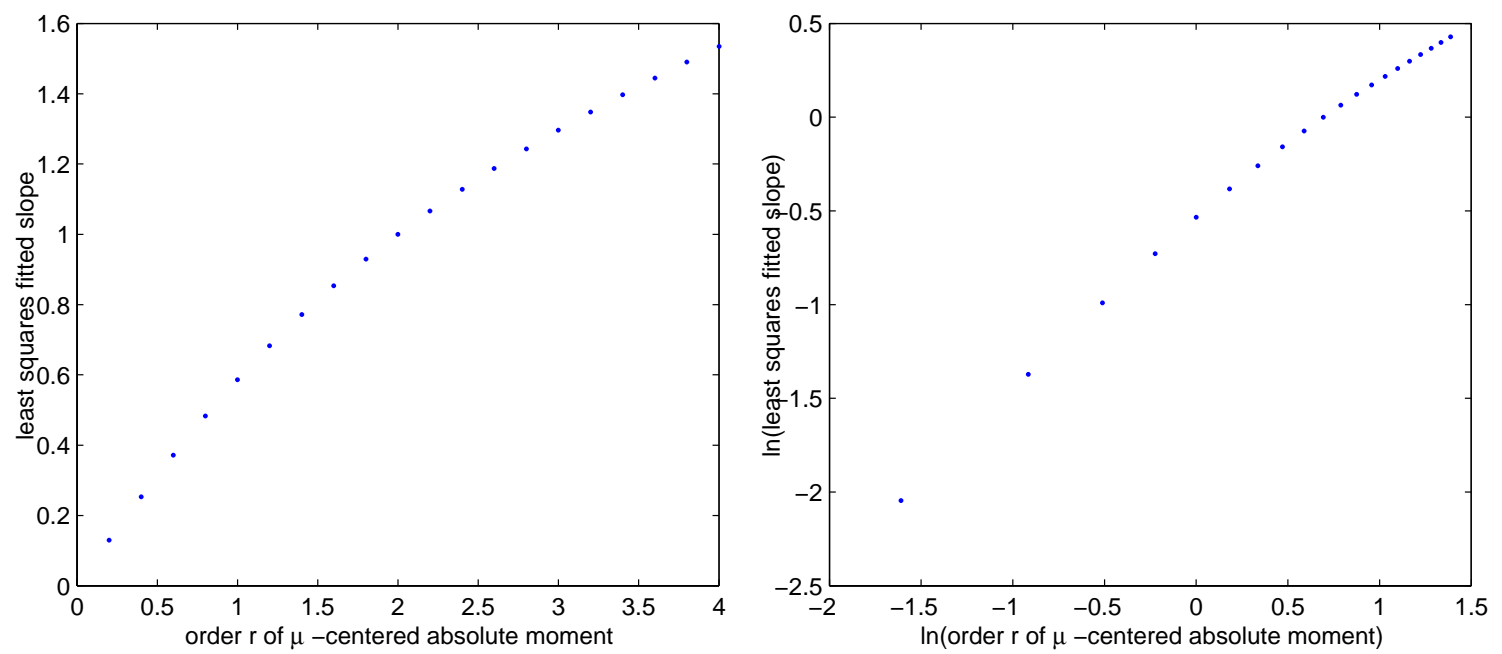

Figure 5: Order of the $\mu$-centered absolute moment of the NIG Lévy process against apparent scaling coefficient and log-log plot

\section{Acknowledgments}

The authors thank Olsen \& Associates, Zürich, for access to the HFDF96 data set via the Centre for Analytical Finance $(\mathrm{CAF})$ at the University of Århus and the Århus School of Business, which is funded by the Danish Social Science Foundation.

This paper was written while the second author was visiting the Department of Mathematical Sciences at the University of Århus under the European Union's Socrates/Erasmus programme. He would like to thank for the pleasant hospitality extended to him.

Corresponding Author:

Robert Stelzer

Lehrstuhl für Mathematische Statistik M4

Zentrum Mathematik

Technische Universität München

Boltzmannstr. 3

D- 85747 Garching bei München

Germany

Email: rstelzer@ma.tum.de

\section{A Moments of GIG and normal laws}

For completeness we provide below the well-known formulae for the moments of the GIG and normal laws. 
For the GIG law the following result is given in Jørgensen (1982, p. 13), who uses a slightly different parametrization.

Lemma 12 Let $X \sim G I G(\nu, \delta, \gamma)$ with $\delta, \gamma>0$. Then

$$
E\left(X^{r}\right)=\left(\frac{\delta}{\gamma}\right)^{r} \frac{K_{\nu+r}(\bar{\gamma})}{K_{\nu}(\bar{\gamma})}
$$

for every $r>0$.

\section{Proof:}

$$
\begin{aligned}
E\left(X^{r}\right) & =\int_{0}^{\infty} \frac{\bar{\gamma}^{\nu}}{2 K_{\nu}(\bar{\gamma})} \delta^{-2 \nu} x^{\nu+r-1} \exp \left(-\frac{1}{2} \bar{\gamma}\left(\left(\bar{\gamma} \delta^{-2} x\right)^{-1}+\bar{\gamma} \delta^{-2} x\right)\right) d x \stackrel{y:=\bar{\gamma}^{-2} x}{=} \\
& =\frac{\bar{\gamma}^{-r} \delta^{2 r}}{K_{\nu}(\bar{\gamma})} \frac{1}{2} \int_{0}^{\infty} y^{\nu+r-1} \exp \left(-\frac{1}{2} \bar{\gamma}\left(y^{-1}+y\right)\right) d y=\left(\frac{\delta}{\gamma}\right)^{r} \frac{K_{\nu+r}(\bar{\gamma})}{K_{\nu}(\bar{\gamma})}
\end{aligned}
$$

where in the last step we employed the integral representation of $K_{\nu+r}$ stated earlier when introducing the modified Bessel function of the third kind (equation (2)).

The absolute moments of the normal distribution $N(0,1)$ are well known and given in many standard texts on probability theory, viz.:

Lemma 13 Let $X \sim N(0,1)$ and $r>0$ then

$$
E\left(|X|^{r}\right)=\frac{2^{r / 2} \Gamma\left(\frac{r+1}{2}\right)}{\sqrt{\pi}} .
$$

Proof:

$$
\begin{aligned}
E\left(|X|^{r}\right) & =(2 \pi)^{-1 / 2} \int_{\mathbb{R}}|x|^{r} e^{-\frac{x^{2}}{2}} d x=\left(\frac{2}{\pi}\right)^{1 / 2} \int_{0}^{\infty} x^{r} e^{-\frac{x^{2}}{2}} d x \stackrel{t:=\frac{x^{2}}{2}}{=} \\
& =\frac{2^{r / 2}}{\sqrt{\pi}} \int_{0}^{\infty} t^{\frac{r+1}{2}-1} e^{-t} d t=\frac{2^{r / 2}}{\sqrt{\pi}} \Gamma\left(\frac{r+1}{2}\right)
\end{aligned}
$$

\section{References}

Barndorff-Nielsen, O. E.: 1977, Exponentially decreasing distributions for the logarithm of particle size, Proceedings of the Royal Society of London A 353, 401419 .

Barndorff-Nielsen, O. E.: 1978a, Hyperbolic distributions and distributions on hyperbolae, Scandinavian Journal of Statistics 5, 151-157.

Barndorff-Nielsen, O. E.: 1978b, Information and Exponential Families in Statistical Theory, John Wiley and Sons, Chichester.

Barndorff-Nielsen, O. E.: 1997, Normal inverse Gaussian distributions and stochastic volatility modelling, Scandinavian Journal of Statistics 24, 1-13. 
Barndorff-Nielsen, O. E.: 1998a, Probability and Statistics; selfdecomposability, finance and turbulence, in L. Accardi and C. C. Heyde (eds), Proceedings of the Conference "Probability towards 2000" held at Columbia University, New York, 2-6 October 1995, Springer, Berlin, pp. 47-57.

Barndorff-Nielsen, O. E.: 1998b, Processes of normal inverse Gaussian type, Finance and Stochastics 2, 41-68.

Barndorff-Nielsen, O. E.: 2001, Modelling by Lévy processess, in I. V. Basawa, C. C. Heyde and R. L. Taylor (eds), Selected Proceedings of the Symposium on Inference for Stochastic Processes, Vol. 37 of Lecture Notes - Monograph Series, Institute of Mathematical Statistics, Hayworth, California, pp. 25-31.

Barndorff-Nielsen, O. E., Blæsild, P. and Halgreen, C.: 1978, First hitting time models for the generalized inverse Gaussian distribution, Stochastic Processes and Applications 7, 49-54.

Barndorff-Nielsen, O. E., Blæsild, P. and Schmiegel, J.: 2004, A parsimonious and universal description of turbulent velocity increments, Research Report 2004-4, MaPhySto, Department of Mathematical Sciences, University of Aarhus.

Barndorff-Nielsen, O. E., Kent, J. T. and Sørensen, M.: 1982, Normal variance-mean mixtures and $z$ distributions, International Statistical Review 50, 145-159.

Barndorff-Nielsen, O. E. and Prause, K.: 2001, Apparent scaling, Finance and Stochastics 5, 103-113.

Barndorff-Nielsen, O. E. and Shephard, N.: 2001, Modelling by Lévy processess for financial econometrics, in O. E. Barndorff-Nielsen, T. Mikosch and S. I. Resnick (eds), Lévy Processes - Theory and Applications, Birkhäuser, Basel, pp. 283318.

Barndorff-Nielsen, O. E. and Shephard, N.: 2005, Continuous Time Approach to Financial Volatility, Cambridge University Press. To appear.

Bibby, B. M. and Sørensen, M.: 1997, A hyperbolic diffusion model for stock prices, Finance and Stochastics 1, 25-41.

Bronstein, I., Semendjaev, K., Mühlig, H. and Musiol, G.: 2000, Taschenbuch der Mathematik, 5th edn, Verlag Harri Deutsch, Frankfurt am Main.

Cont, R. and Tankov, P.: 2004, Financial Modeling with Jump Processes, Chapman \& Hall/CRC, London.

Eberlein, E.: 2001, Application of generalized hyperbolic Lévy motions to finance, in O. E. Barndorff-Nielsen, T. Mikosch and S. I. Resnick (eds), Lévy Processes - Theory and Applications, Birkhäuser, Basel, pp. 320-336.

Eberlein, E. and Hammerstein, E. A. v.: 2002, Generalized hyperbolic and inverse Gaussian distribution: Limiting cases and approximation of processes, FDM Preprint 80, Department of Mathematical Stochastics, University of Freiburg.

Eberlein, E. and Keller, U.: 1995, Hyperbolic distributions in finance, Bernoulli 1, 281-299. 
Emmer, S. and Klüppelberg, C.: 2004, Optimal portfolios when stock prices follow an exponential Lévy process, Finance and Stochastics 8, 17-44.

Freitag, E. and Busam, R.: 2000, Funktionentheorie I, 3rd edn, Springer, Berlin.

Gradshteyn, I. and Ryzhik, I. W.: 1965, Tables of Integrals, Series and Products, Academic Press, New York.

Guillaume, D. M., Dacorogna, M. M., Davé, R. D., Müller, U. A., Olsen, R. B. and Pictet, O. V.: 1997, From the bird's eye to the microscope: a survey of new stylized facts of the intra-daily foreign exchange markets, Finance and Stochastics 1, 95-129.

Ismail, M. E. H.: 1977, Integral representations and complete monotonicity of various quotients of Bessel functions, Canadian Journal of Mathematics 29, 1198-1207.

Jørgensen, B.: 1982, Statistical Properties of the Generalized Inverse Gaussian Distribution, Lecture Notes in Statistics, Springer, Heidelberg.

Müller, U. A., Dacorogna, M. M., Olsen, R. B., Pictet, O. V., Schwarz, M. and Morgenegg, C.: 1990, Statistical study of foreign exchange rates, empirical evidence of a price scaling law and intraday analysis, Journal of Banking and Finance 14, 1189-1208.

Prause, K.: 1997, Modelling financial data using generalized hyperbolic distributions, FDM Preprint 48, Department of Mathematical Stochastics, University of Freiburg.

Prause, K.: 1999, The Generalized Hyperbolic Model: Estimation, Financial Derivatives and Risk Measures, Dissertation, Mathematische Fakultät, AlbertLudwigs-Universität Freiburg im Breisgau.

Raible, S.: 2000, Lévy Processes in Finance: Theory, Numerics and Empirical Facts, Dissertation, Mathematische Fakultät, Albert-Ludwigs-Universität Freiburg im Breisgau.

Rydberg, T. H.: 1997, The normal inverse Gaussian Lévy process: Simulation and approximation, Communication in Statistics: Stochastic Models 13, 887-910.

Rydberg, T. H.: 1999, Generalized hyperbolic diffusions with applications towards finance, Mathematical Finance 9, 183-201.

Samorodnitsky, G. and Taqqu, M. S.: 1994, Stable Non-Gaussian Random Processes, Stochastic Modeling, Chapman \& Hall/CRC, Boca Raton.

Sato, K.-I.: 1999, Lévy Processes and Infinitely Divisible Distributions, Cambridge Studies in Advanced Mathematics, Cambridge University Press.

Schoutens, W.: 2003, Lévy Processes in Finance - Pricing Financial Derivatives, Wiley Series in Probability and Statistics, John Wiley and Sons, Chichester.

Watson, G. N.: 1952, A Treatise on the Theory of Bessel Functions, reprinted 2nd edn, Cambridge University Press. 